\title{
PKC- $\alpha$ Triggers EGFR Ubiquitination, Endocytosis and ERK Activation in Podocytes Stimulated with High Glucose
}

\author{
Chun-Tao Lei Yan-Hong Wei Hui Tang Qian Wen Chen Ye Chun Zhang \\ Hua Su
}

Department of Nephrology, Union Hospital, Tongji Medical College, Huazhong University of Science and Technology, Wuhan, China

\section{Key Words}

PKC- $\alpha \cdot E G F R \cdot$ Ubiquitination • Endocytosis • Podocyte

\begin{abstract}
Background: Protein Kinase C- $\alpha$ (PKC- $\alpha$ ) and epidermal growth factor receptor (EGFR) are both involved in diabetic kidney disease; however, the connection between these two proteins during high glucose-induced podocyte injury remains uncertain. Methods: Diabetes was induced in SD rats by streptozotocin (STZ). Fourteen days later, the kidney cortex was removed and subjected to plasma membrane isolation and lipid raft fractionation. In vitro study human podocyte cell line was differentiated and subjected to various treatments. The levels of membranous protein and endocytosis were assessed by biotinylation and sodium 2-mercaptoethane sulfonate (MesNa) treatment. Gö6976 and PYR-41 were used as inhibitors of PKC- $\alpha$ and ubiquitin activating E1 enzyme, respectively. Results: In diabetic rats, the abundance of PKC- $\alpha$ in the membranous fraction and the lipid raft domain is elevated, whereas the EGFR level is reduced. Consistently, in vitro high glucose treated podocytes, membranous EGFR is downregulated with increased PKC- $\alpha$. Furthermore, the ubiquitination and endocytosis of EGFR are enhanced accompanied by extracellular signal-regulated kinase (ERK) signaling activation and podocyte damage during hyperglycemia. However, these processes can be ameliorated by inhibition of either PKC- $\alpha$ or ubiquitin activating E1 enzyme. Conclusion: During hyperglycemia, PKC- $\alpha$ mediates podocytic EGFR ubiquitination, endocytosis from cell surface and the subsequent ERK activation, which contributes to podocyte injury.
\end{abstract}

(C) 2017 The Author(s)

Published by S. Karger AG, Basel

\section{Introduction}

The global epidemic of diabetes has resulted in a continuously increasing prevalence of diabetic kidney disease (DKD) in recent decades. Currently, DKD is the leading cause of endstage renal disease worldwide. Effective treatment options are needed to reduce the social and economic burden of DKD, and a detailed understanding of the pathogenesis of DKD is critical for developing successful management strategies.

C.-T. Lei, Y.-H. Wei and H. Tang contributed equally to this work. 


\section{Cellular Physiology Cell Physiol Biochem 2017;42:281-294 \begin{tabular}{l|l} 
DOI: 10.1159/000477329 & O 2017 The Author(s). Published by S. Karger AG, Basel \\
www.karger.com/cpb
\end{tabular} \\ Lei et al.: PKC- $\alpha$-EGFR-ERK Axis Involves in High Glucose Induced Podocyte Injury}

The protein kinase C (PKC) superfamily is involved in many physiological processes and signaling events and comprises three subfamilies: conventional PKCs (PKC- $\alpha, \beta, \gamma$ ), novel PKCs and atypical PKCs. In diabetes, the PKC superfamily is extensively activated by various pathogenic stimuli $[1,2]$. Each isoform of the PKC subfamilies has a specific function or activation mechanism, and the different isoforms exhibit specific, non-redundant biological functions that cannot be compensated for by other isoforms [3]. It is well documented that PKC- $\alpha$ is the primary PKC superfamily isoform involved in DKD, while PKC- $\beta$ is primarily involved in immune-mediated glomerulonephritis, includinglupusnephritisandmembranous proliferative glomerulonephritis [4]. PKC- $\alpha$ knockout mice show reduced albuminuria and attenuated nephrin loss in a streptozotocin (STZ)-induced model of diabetes. Conversely, the genetic deletion of PKC- $\beta$ in mice does not preserve nephrin expression in diabetic mice, although it does attenuate extracellular matrix (ECM) accumulation in the mesangial region [5-7]. Thus, PKC- $\alpha$ is the dominant isoform of PKC-associated podocyte injury in diabetes mellitus.

Epidermal growth factor receptor (EGFR) is a membrane protein that belongs to the ErbB family of receptor tyrosine kinases. After activation, EGFR is internalized from the cell surface and stimulates diverse signaling cascades, leading to a variety of pathophysiological processes, such as cell lineage and fate determination, proliferation, and migration [8]. EGFR has been well studied in the field of cancer research. In the kidney, EGFR signaling contributes to podocyte transdifferentiation and conversion to a proliferative and migratory phenotype during rapidly progressive crescentic glomerulonephritis in mice and humans [9, 10]; however, it is not indispensible for effacement of podocyte foot processes and urinary protein loss in lipopolysaccharide-induced proteinuria [10]. These findings show that EGFR exerts distinct functions in different disorders that are closely related to the underlying etiology and pathogenesis of renal disorders. Of note, as a membrane protein, the trafficking of EGFR determines the subsequent signaling transduction in a stimulus and cell contextdependent fashion [10-12].

In the past few decades, several studies in the field of tumorigenesis have illustrated that PKC- $\alpha$ regulates EGFR activation. Nevertheless, the conclusions are controversial. In a mouse model of multiple intestinal neoplasias and in human squamous carcinoma and colon carcinoma cell lines, PKC- $\alpha$ has been shown to abrogate EGFR signal transduction [13-15], while in a human prostate cancer cell line, PKC- $\alpha$ stimulates EGFR signaling [16]. Thus, we are interested in exploring how PKC- $\alpha$ modulates EGFR trafficking and signaling in diabetes, especially in podocytes, a critical component of DKD. The experiments outlined in the current study were designed specifically to explore the association between PKC- $\alpha$ and EGFR trafficking and signal transduction in podocytes in hyperglycemia. We found that high glucose (HG) induces the movement of PKC- $\alpha$ to the cell surface, which contributes to the subsequent EGFR ubiquitination and internalization, extracellular signal-regulated kinase (ERK) activation and, ultimately, podocyte injury. Blocking the activity of PKC- $\alpha$ or ubiquitinactivating enzyme eventually attenuates EGFR internalization, ERK phosphorylation and podocyte damage.

\section{Materials and Methods}

\section{Animal model}

Animal experiments were carried out in accordance with the Guidelines for the Care and Use of Laboratory Animals of the National Institutes of Health (NIH) and were approved by the Animal Care and Use Committee (ACUC) of Tongji Medical College, Huazhong University of Science and Technology. Male Sprague-Dawley rats (Charles River Laboratories, Wilmington, MA) weighing 125-200 g were given free access to clean water and standard rat chow. Rats were injected with STZ (Sigma, St. Louis, MO; $62.5 \mathrm{mg} / \mathrm{kg}$ body wt prepared freshly in $0.1 \mathrm{M}$ citrate buffer, $\mathrm{pH} 4.0$ ) or a vehicle control via a tail vein to establish the STZ-induced type 1 diabetic rat model, as previously reported. Hyperglycemia was verified 2 days after the 


\section{Cellular Physiology Cell Physiol Biochem 2017;42:281-294

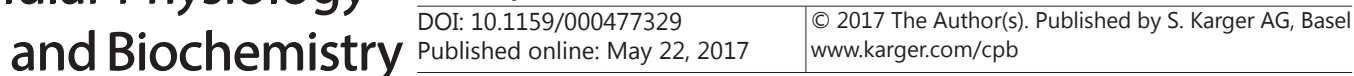

Lei et al.: PKC- $\alpha$-EGFR-ERK Axis Involves in High Glucose Induced Podocyte Injury

injection using a LifeScan Ultra II glucometer. Fourteen days after the injection, the kidneys were removed; the cortices were isolated, homogenized with glass homogenizers in a modified radioimmunoprecipitation assay (RIPA) buffer (150 mM NaCl, $10 \mathrm{mM}$ Tris $\cdot \mathrm{HCl}$ (pH 7.5), $1 \mathrm{mM}$ EDTA, 1\% Triton X-100, 1\% sodium deoxycholate, $0.1 \%$ SDS, and protease inhibitors) and centrifuged at $8,000 \mathrm{~g}$ for $12 \mathrm{~min}$. The protein concentration of the supernatant fractions was measured using BCA Protein Assay kits (Pierce). The lysates were processed for the following experiments.

Plasma membrane and lipid raft fractionation

Plasma membranes (PMs) from tissue lysates and podocytes were prepared via5-step sucrosegradient $(2.0,1.6,1.4,1.2$, and $0.8 \mathrm{M})$ ultracentrifugation, as previously described [17]. The fraction at the interface of the 1.6 and $1.4 \mathrm{M}$ densities was collected as the PM fraction.

The lipid raft microdomain was isolated with a 5-40\% discontinuous sucrose gradient, as previously described [17]. Briefly, rat cortex suspensions were prepared and lysed in ice-cold 0.5\% Brij 96-TNEV buffer for 30 min on ice. Five hundred microliters of postnuclear supernatant were mixed with an equal volume of $80 \%$ sucrose in TNEV buffer and transferred to $13 \times 51 \mathrm{~mm}$ Beckman centrifuge tubes. Three milliliters of $35 \%$ sucrose in TNEV were layered carefully on top of the mixture followed by another 1 ml layer of 5\% sucrose. The sucrose gradient was centrifuged in a SW 50.1 rotor (Beckman, Brea, CA, USA) at 34,000 rpm $(110,000 \mathrm{~g})$ at $4^{\circ} \mathrm{C}$ for $18 \mathrm{~h}$. Equal volumes of 13 fractions (approximately $400 \mu \mathrm{l}$ ) were collected from the top to the bottom of the tube and assessed via immunoblotting with the appropriate antibodies. Caveolin-1 was used as a marker for lipid rafts.

Cell culture and treatment

An immortalized human podocyte cell line was routinely cultured, as described in our previous publication [18]. After the cells reached $85 \%$ confluence at $33^{\circ} \mathrm{C}$, they were shifted to $37^{\circ} \mathrm{C}$ for $10-14$ days to allow for differentiation. Then, the cells were exposed to the different test compounds alone or in combination, as described in detail in the relevant experiments. Cells were then subjected to subcellular organelle isolation, immunoprecipitation, cell surface biotinylation, internalization and Western blot analyses. The well-accepted PKC- $\alpha$ inhibitor Gö6976 [19, 20] was purchased from Tocris Bioscience (Cat. No. 2253). PYR-41 was obtained from Calbiochem (Cat. No. 662105).

Cell surface biotinylation and endocytosis assays

Cell surface protein expression was evaluated using the membrane-impermeable biotinylation reagent NHS-SS-biotin, as previously described [21]. Briefly, cells were seeded on 6-well plates and cultured with $1.2 \mathrm{ml}$ of NHS-SS-biotin $(0.6 \mathrm{mg} / \mathrm{ml}$ in a borate buffer $)$ in two successive 20 -min incubations on ice with very gentle shaking. Each time, the reagent was freshly prepared and placed on ice. After biotinylation, each well was rinsed briefly with $2 \mathrm{ml}$ of quenching solution (100 mM glycine in PBS) and then incubated with the same solution for $10 \mathrm{~min}$ on ice to ensure complete neutralization of uncrosslinked NHS-SS-biotin. The cells were then lysed on ice with $600 \mathrm{ml}$ of lysis buffer. The cell lysates were centrifuged at $16,000 \mathrm{~g}$ at $4^{\circ} \mathrm{C}$. Then, $30 \mu \mathrm{l}$ of streptavidin-agarose beads were added to the supernatant, and the samples were rotated in a cold room overnight. The next day, after centrifugation at 3,000 $\mathrm{g}$ for $1 \mathrm{~min}$, the supernatant was subject to immunoblotting to examine the cell membrane proteins.

EGFR internalization was measured via biotinylation and sodium 2-mercaptoethane sulfonate (MesNa) treatment [21]. In short, the membranous proteins on the cell surface were first biotin-labeled with a freshly prepared solution of $0.6 \mathrm{mg} / \mathrm{ml} \mathrm{EZ-Link} \mathrm{sulfo-NHS-SS-biotin} \mathrm{(Pierce} \mathrm{21331)} \mathrm{in} \mathrm{a} \mathrm{borate} \mathrm{buffer} \mathrm{as} \mathrm{described}$ above. The biotin reaction was quenched for $10 \mathrm{~min}$ with $0.1 \mathrm{M}$ lysine (Sigma). After they were washed with PBS, cells were added to pre-warmed culture medium (without or with the different treatment compounds) and incubated at $37^{\circ} \mathrm{C}$ for the indicated time period to allow for protein endocytosis. Control cells were kept on ice to prevent endocytosis. The non-internalized biotin was cleaved 3 times with the cell-impermeable reducing agent MesNa (50 mM MesNa, $1 \mathrm{mM}$ EDTA, 0.2\% BSA in $50 \mathrm{mM}$ Tris (pH 8.6) for 20 min at $4^{\circ} \mathrm{C}$ on a rocking platform. The biotin bound to the endocytosed proteins was protected from MesNa cleavage. After they were quenched with iodoacetamide and subsequently washed in PBS, cells were solubilized in RIPA buffer. The biotinylated proteins were retrieved by streptavidin-agarose affinity precipitation, and the endocytosed EGFR was detected via Western blotting with an EGFR antibody.

\section{KARGER}




\section{Cellular Physiology Cell Physiol Biochem 2017;42:281-294 \begin{tabular}{l|l|l} 
and Biochemistry.1159/000477329 & $\begin{array}{l}\text { (c) } 2017 \text { The Author(s). Published by S. Karger AG, Basel } \\
\text { www.karger.com/cpb }\end{array}$
\end{tabular} \\ Lei et al.: PKC- $\alpha$-EGFR-ERK Axis Involves in High Glucose Induced Podocyte Injury}

Cell lysate preparation, immunoprecipitation and Western blotting

After treatment, cells were lysed in RIPA buffer, and the protein concentration of the cleared lysate was determined using BCA Protein Assay kits (Pierce). For immunoprecipitation, equal amounts (0.5-1 mg) of total protein samples were incubated with EGFR antibodies at $4^{\circ} \mathrm{C}$ overnight with gentle mixing, followed by the addition of $10 \mu \mathrm{l}$ of protein $\mathrm{A} / \mathrm{G}$ beads (Pierce) and continued incubation for another $2 \mathrm{~h}$. The beads were pelleted via centrifugation at 3,000 rpm for $1 \mathrm{~min}$ and washed three times with RIPA buffer. The precipitated proteins were eluted in $40 \mu \mathrm{l}$ of Laemmli sample buffer. For Western blot analyses, the proteins were separated via 4-15\% SDS-PAGE and electrotransferred to polyvinylidene difluoride membranes (BioRad). The membranes were routinely processed via blocking with $5 \%$ milk and were incubated overnight with primary antibodies, followed by a $1 \mathrm{~h}$ incubation with horseradish peroxidase-conjugated secondary antibodies. Immunoreactive proteins were detected using an Enhanced Chemiluminescence (ECL) Kit (Amersham). The antibodies used in this study were as follows: polyclonal rabbit EGFR antibody (1:100; Santa Cruz Biotechnology, Santa Cruz, CA, USA), monoclonal mouse Ub antibody (1:100; P4D1, Santa Cruz Biotechnology), polyclonal rabbit p-ERK antibody, monoclonal mouse desmin antibody (1:1000; Protein Tech Group, Chicago, IL, USA), and monoclonal mouse $\beta$-actin antibody (1:10000; Santa Cruz Biotechnology).

Staining of F-actin

F-actin staining was performed as described previously [22,23]. Briefly, podocytes were cultured in 24well plates the day before treatment to assess the effect of ubiquitination on their cytoskeletal arrangement, which is basically composed of F-actin. The next day, podocytes were exposed to HG for $24 \mathrm{~h}$ without or with PYR-41 pretreatment. Then, the cells were fixed in 4\% paraformaldehyde and permeabilized with $0.1 \%$ Triton X-100. F-actin was stained with rhodamine-phalloidin (Invitrogen Corp., Carlsbad, CA, USA) for 15 $\mathrm{min}$ at room temperature. The slides were observed under a confocal laser scanning microscope at identical settings. The cells with well-organized, distinct, longitudinal F-actin fibers were recognized as undamaged and counted, and scoring was obtained from 80 podocytes on each slide in the different groups to calculate the percentage of normal cells.

\section{Statistical analysis}

The data displayed on the graphs are mean values, with error bars representing the standard error of the mean (SEM). Significant differences among multiple groups were determined using an analysis of variance (ANOVA) followed by Student-Newman-Keuls post hoc tests, and $\chi^{2}$ tests were used to test the significance of the data expressed as ratios and percentages. $P<0.05$ was considered to indicate statistical significance.

\section{Results}

The abundance of membranous PKC- $\alpha$ and EGFR in the renal cortices of STZ-induced diabetic rats

Renal cortex PMs were isolated via 5-step sucrose-gradient ultracentrifugation. The whole tissue lysates (WTLs) and PM fractions were immunoblotted with PKC- $\alpha$ and EGFR antibodies. In STZ-induced type 1 diabetic rats, the level of membranous PKC- $\alpha$ was dramatically elevated, whereas the abundance of the EGFR protein at the cell surface was markedly decreased (Fig. 1 a, b). Furthermore, to specify the microdomain in which PKC- $\alpha$ and EGFR aggregated, we employed a lipid raft isolation technique. We found that in control rats, a large quantity of EGFR protein was located in the lipid raft domain, but only low levels of PKC- $\alpha$ were detected. In contrast, in the renal cortices of diabetic rats, a large quantity of PKC- $\alpha$ protein was detected in the lipid raft domain, accompanied by a rather weak EGFR signal intensity (Fig. 1c).

The expression of membranous PKC- $\alpha$ and EGFR in cultured human podocytes with HG stimulation

Next, we evaluated whether HG exposure affects the quantity of membranous PKC- $\alpha$ and EGFR in cultured human podocytes. First, we isolated cell membranes via 5-step sucrosegradient ultracentrifugation. Then, the whole cell lysates (WCLs) and PM fractions were 
Fig. 1. EGFR expression was reduced, and PKC- $\alpha$ expression was increased in the membranous fractions from the cortices of diabetic rats. Fourteen days after a single intra-peritoneal STZ injection, the renal cortex was removed. (a) The tissue lysate was subjected to plasma membrane (PM) isolation via 5-step sucrose-gradient ultracentrifugation. Equal amounts of whole tissue lysates (WTLs) and PMs were immunoblotted with PKC- $\alpha$ and EGFR antibodies. $\beta$-actin and $\mathrm{Na}^{+} / \mathrm{K}^{+}$ATPase were used as internal standards for WTL and PM quantification, respectively. (b) The bar graph shows the quantification of the PKC- $\alpha$ and EGFR signals from the control (Ctrl) and diabetic
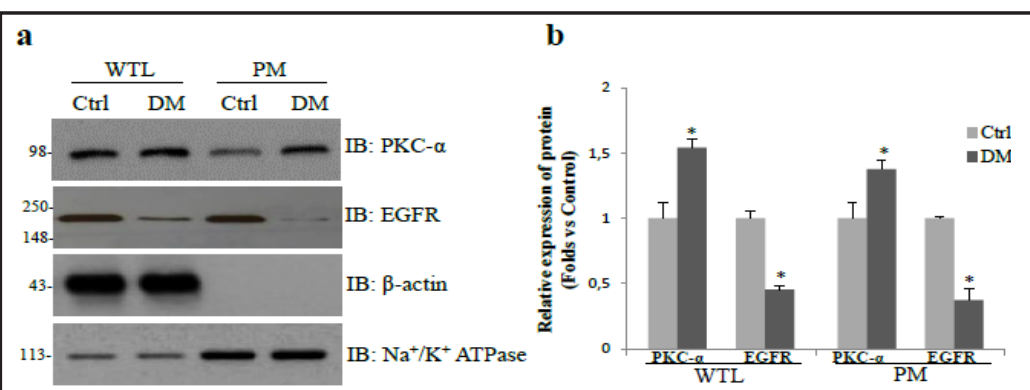

c
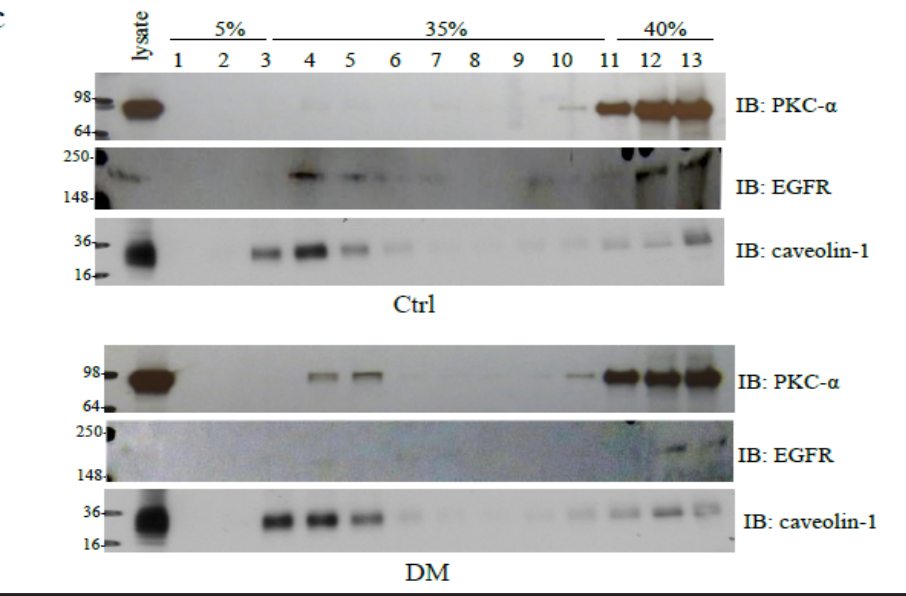

(DM) rat cortices. The relative intensity of protein expression in the Ctrl rats was set to 1 (mean $\pm S E M, n=6$, ${ }^{*} \mathrm{P}<0.05$ vs. Ctrl). (c) Lipid raft experiment. Renal cortex suspensions from rats were prepared and lysed in TENV buffer. The lysate supernatants were loaded on a 5-40\% sucrose gradient, as described in the Materials and Methods section. After $18 \mathrm{~h}$ of ultracentrifugation, equal quantities of the fractions were collected and used for immunoblotting with PKC- $\alpha$ and EGFR antibodies. Caveolin-1 was used as a marker of lipid rafts. Each analysis was performed in triplicate.

immunoblotted with PKC- $\alpha$ and EGFR antibodies. We found that the level of membranous PKC- $\alpha$ increased, while the abundance of membranous EGFR decreased in podocytes exposed to HG. This result is in accordance with data from in vivo studies (Fig. 2 a, b). Moreover, using cell surface biotinylation assays to examine the levels of cell surface proteins, we reconfirmed the above findings and showed that these alterations occur in a time-dependent manner (Fig. 2 c, d).

\section{$H G$-triggered EGFR ubiquitination is PKC- $\alpha$ required in human podocytes}

Many studies have revealed that membranous PKC can mediate cell surface receptors, channel ubiquitination and internalization, and intracellular trafficking, which prompt us to speculate that the upregulated membranous PKC- $\alpha$ may initiate EGFR ubiquitination, regulating its internalization and subsequent intracellular transportation. Using coimmunoprecipitation, we evaluated the ubiquitination of EGFR in podocytes during HG exposure. As shown in Fig. 3 a and b, HG induced podocytic EGFR ubiquitination in a time-dependent manner. Interestingly, pretreatment with the PKC- $\alpha$ inhibitor Gö6976 dramatically diminished EGFR ubiquitination (Fig. 3 c, d), which suggests that HG-mediated EGFR ubiquitination is PKC- $\alpha$-dependent.

$H G$ promotes EGFR endocytosis from the cell surface, which is PKC- $\alpha$ - and ubiquitinationdependent

EGFR is situated on the surface of membranes and can sense stimuli in a ligand-mediated or non-ligand-mediated fashion. Internalization from the cell surface and the post-endocytotic KARGER 
Fig. 2. High glucose (HG) exposure mediates the increase in PKC- $\alpha$ and the reduction in EGFR in the PM fraction of human podocytes. (a) Human podocytes were treated with an osmotic control (Ctrl) $(19.9 \mathrm{mmol} / \mathrm{L}$ of mannitol) or 30 $\mathrm{mmol} / \mathrm{L}$ of glucose (HG) for $48 \mathrm{~h}$ and were then subjected to $\mathrm{PM}$ isolation via 5-step sucrose-gradient ultracentrifugation. The whole cell lysates (WCLs) and PMs were probed for PKC- $\alpha$ and EGFR. $\beta$-actin and $\mathrm{Na}^{+} / \mathrm{K}^{+}$ ATPase were used as
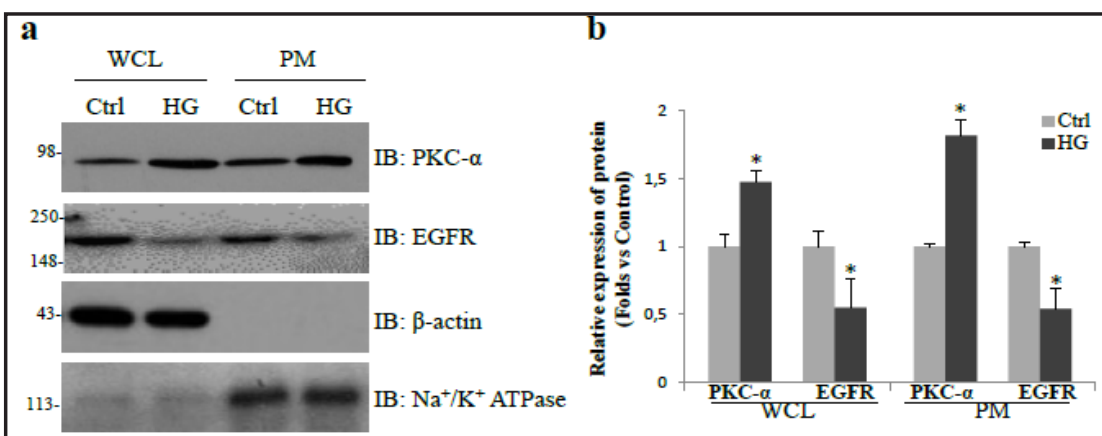
c
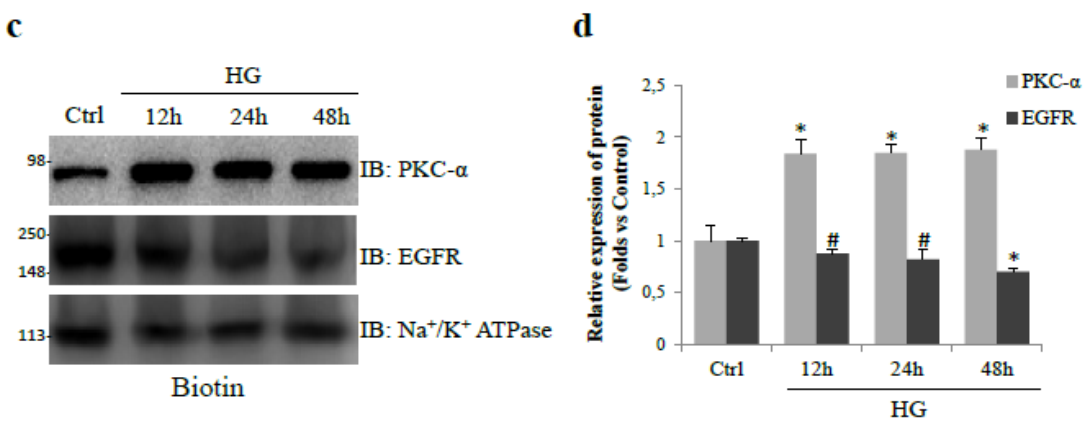
internal controls. (b) Densitometry analysis. The bands were quantified, and the relative density of the cells was determined, with the osmotic control set as a density of $1\left(\mathrm{n}=4,{ }^{*} \mathrm{P}<0.05\right.$ vs. Ctrl). (c) Human podocytes were exposed to Ctrl or HG conditions for the indicated time periods. Cells were then processed for biotinylation assays. The biotinylated samples were immunoblotted with PKC- $\alpha$ and EGFR antibodies. (d) The bands were quantified, and the relative density of the biotinylated proteins in the Ctrl group was set to 1 ( $\mathrm{n}=4,{ }^{*} \mathrm{P}<0.05$ vs. Ctrl; ${ }^{\mathrm{P}} \mathrm{P}>0.05$ vs. Ctrl).

trafficking of EGFR are the core regulatory mechanisms for its activation and downstream signaling transduction. Thus, we evaluated how HG affects EGFR trafficking in podocytes and its link with post-translational ubiquitination. Utilizing cell surface biotinylation, we proved that the PKC- $\alpha$ inhibitor Gö6976 and the ubiquitin-activating enzyme E1 inhibitor PYR-41 can significantly reverse the decline of membranous EGFR (Fig. 4 a, b). As we know, the downregulation of this cell surface protein may be caused by the reduced forward trafficking of cytoplasmic proteins to the membrane or their accelerated endocytosis from the cell surface. Next, we assessed the internalization of membranous EGFR. First, we labeled the cell surface proteins with NHS-SS-biotin on ice. Then, cells were shifted to $37^{\circ} \mathrm{C}$ with different treatments for the indicated time points, and the unendocytosed membranous proteins were stripped away by MesNa. Therefore, the signal we detected represented the internalized membrane proteins. As shown in Fig. $4 \mathrm{c}$ and d, after HG treatment, the internalization of EGFR from the cell surface increased, which was effectively halted by a PKC- $\alpha$ inhibitor or an E1 ubiquitin enzyme inhibitor. These results suggest that HG promotes the endocytosis of EGFR from the cell membrane and that this depends on PKC- $\alpha$-mediated EGFR ubiquitination.

\section{$H G$-induced ERK activation is reversed by an ubiquitin E1 inhibitor}

EGFR internalization is related to ERK activation. The results presented above indicate that the ubiquitin-activating enzyme E1 inhibitor PYR-41 attenuates EGFR endocytosis induced by HG; therefore, we assessed whether PYR-41 could abolish the activation of ERK. As shown in Fig. 5 a and b, HG exposure increases ERK phosphorylation in a time-dependent manner. However, this effect was markedly suppressed by pretreatment with PYR-41 (Fig. $5 \mathrm{c}, \mathrm{d})$. 
Fig. 3. PKC- $\alpha$ contributes to HG-triggered EGFR ubiquitination in podocytes (a) HG induces EGFR ubiquitination in human podocytes in a time-dependent manner. Human podocytes were exposed to an osmotic control (Ctrl) or $30 \mathrm{mmol} / \mathrm{L}$ of glucose (HG) for the indicated time points. Ubiquitinated EGFR was examined via EGFR immunoprecipitation followed by immunoblotting with an ubiquitin antibody. (b) The bands were quantified. The level of ubiquitinated EGFR was normalized to the immunoprecipitated EGFR. The relative

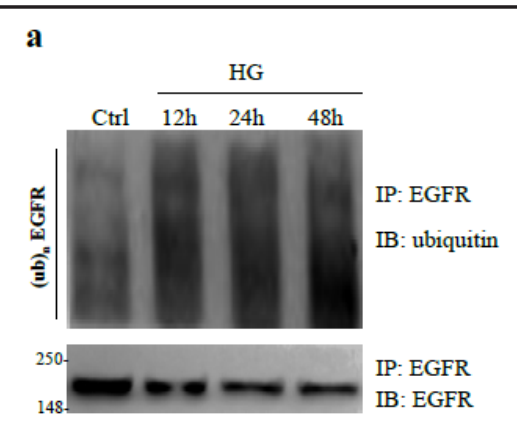
b
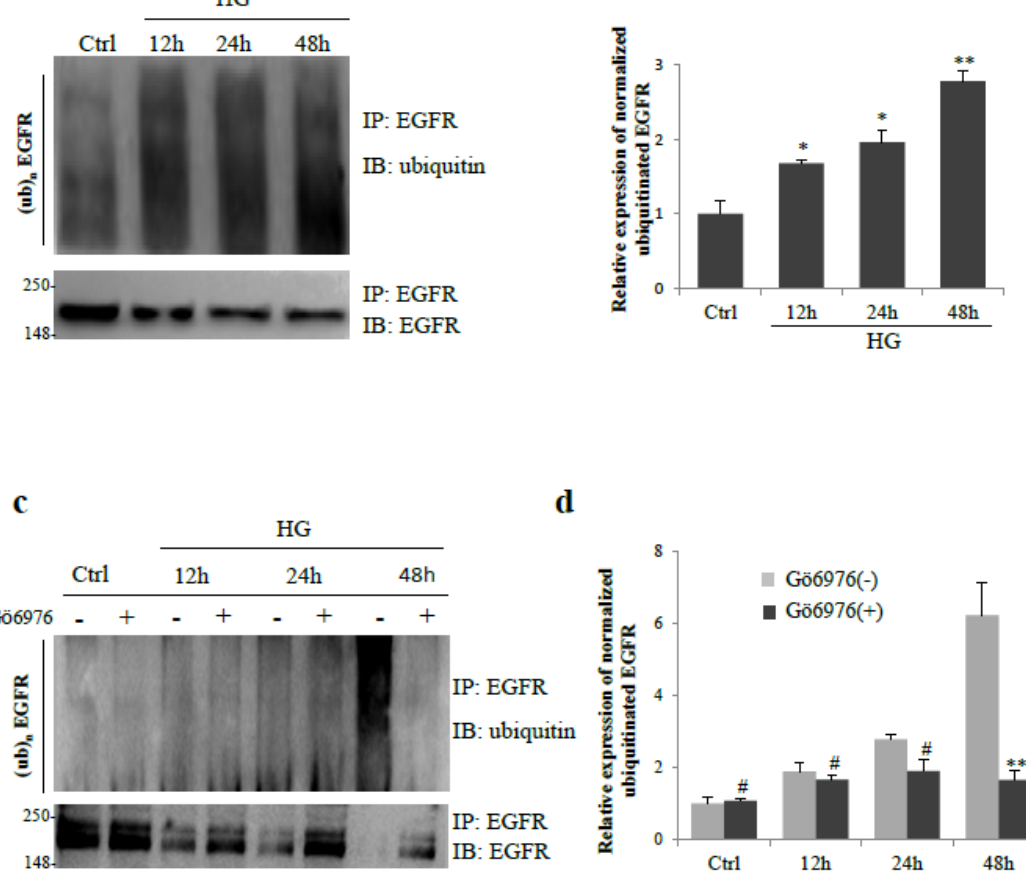

d

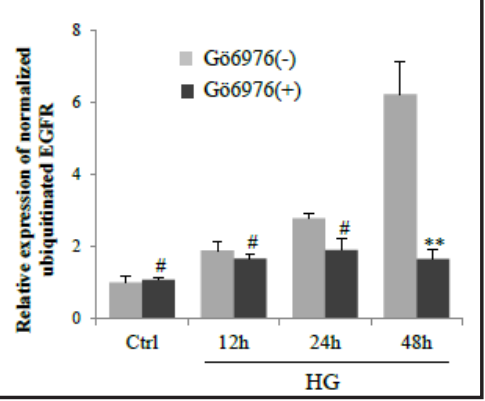
density of the Ctrl group was set to 1 ( $\mathrm{n}=4,{ }^{*} \mathrm{P}<0.05$ vs. Ctrl; ${ }^{* *} \mathrm{P}<0.01$ vs. Ctrl). (c) A PKC- $\alpha$ inhibitor reduces EGFR ubiquitination in podocytes treated with HG. Human podocytes were preincubated with or without $10 \mu \mathrm{M}$ Gö6976 for 10 min and were then subjected to HG stimulation for the indicated time periods. Ubiquitinated EGFR was examined as described above. (d) The normalized level of ubiquitinated EGFR was quantified. The relative intensity of Ctrl group without the Gö6976 treatment was set to $1\left(\mathrm{n}=4,{ }^{* *} \mathrm{P}<0.01 v s\right.$. Gö6976 (-); \#P >0.05 vs. Gö6976 (-)).

\section{Ubiquitin E1 inhibitor attenuates $H G$-induced podocyte damage}

Next, we examined whether blocking the ubiquitination process could prevent podocyte injury during HG exposure. Desmin is a cytoskeletal protein and a marker of podocyte dedifferentiation and damage [24]. As seen in Fig. 6 a-d, the increase in desmin levels under HG stimulation was attenuated by PYR-41 pretreatment. In addition, the blocking of ubiquitination by PYR-41 rescued cells from the HG-induced rearrangement and loss of F-actin, which is an indispensable component for maintaining the specific structure and function of podocytes (Fig. 6 e, f).

\section{Discussion}

This study is the first to report that in diabetic rats, renal PKC- $\alpha$ accumulates on the cell surface and in the lipid raft microdomain and that this accumulation is accompanied by decreased levels of membranous EGFR. Moreover, in HG-treated podocytes, PKC- $\alpha$ aggregates in the membrane fraction and mediates EGFR ubiquitination, which is responsible for membranous EGFR internalization and ERK activation, leading to podocyte damage.

DKD has become one of the most challenging global health issues. Thus, effective therapeutic strategies that target its underlying pathogenesis are urgently required. The characteristic histopathological changes associated with DKD include a reduced number of podocytes in the early stage of the disease (even earlier than the manifestation of albuminuria) and glomerulosclerosis in the advanced stage. Podocytes are terminally differentiated cells that are anchored to the outside of the glomerular capillaries, and they normally prevent the 
Fig. 4. Inhibition of the ubiquitin E1 enzyme and PKC- $\alpha$ prevents HG-induced EGFR endocytosis. Human podocytes were treated with HG for $48 \mathrm{~h}$ alone (Veh) or together with a pretreatment of 10 $\mu \mathrm{M}$ Gö6976 for $10 \mathrm{~min}$ or $10 \mu \mathrm{M}$ PYR-41 for $30 \mathrm{~min}$. (a) Biotinylation experiment. The membranous abundance of EGFR was evaluated via biotinylation analysis. $\mathrm{Na}^{+} / \mathrm{K}^{+}$ATPase was used as an internal standard for membranous protein quantification. (b) Biotinylated EGFR was quantified. The relative intensity of the osmotic control (Ctrl) group was set to

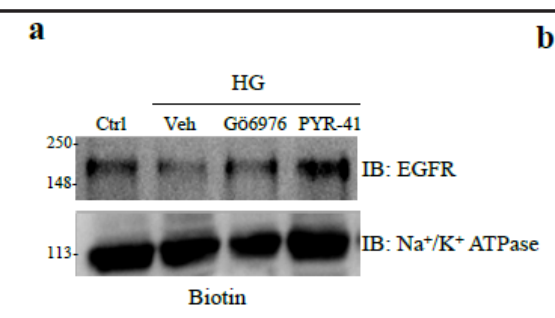

b

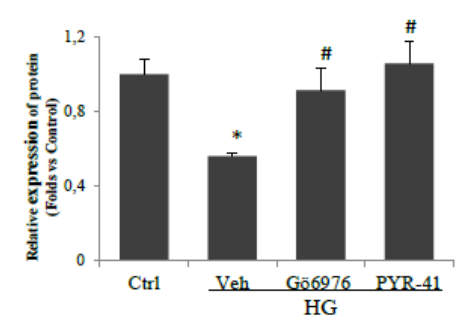

c

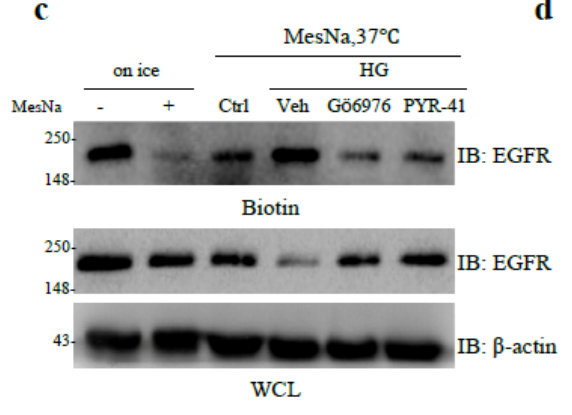

d

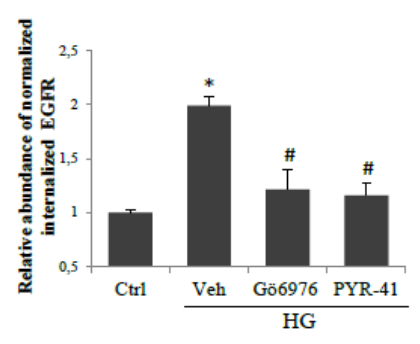

$1\left(\mathrm{n}=4, * \mathrm{P}<0.05\right.$ vs. Ctrl; ${ }^{\mathrm{P}}<0.05$ vs. HG+Veh). (c) EGFR internalization assay. Podocytes were first biotinylated and then warmed to $37^{\circ} \mathrm{C}$ under different treatment conditions. The non-internalized biotin on the cell surface was stripped away with sodium 2-mercaptoethane sulfonate (MesNa). The cells were lysed in RIPA buffer, and the whole cell lysates (WCLs) were used for Western blotting with EGFR and actin antibodies. Internalized proteins were recovered using streptavidin beads and were processed for immunoblotting with an EGFR antibody. (d) The bands were quantified. The internalized EGFR was normalized to the EGFR level in the WCL. The relative density of the Ctrl group was set to 1 ( $\mathrm{n}=3$, ${ }^{*} \mathrm{P}<0.05$ vs. Ctrl; ${ }^{\mathrm{P}}<0.05$ vs. HG+Veh).

Fig. 5. Blocking the ubiquitin E1 enzyme ameliorates HG-mediated ERK activation. (a) Human podocytes were incubated without (Ctrl) or with HG for the indicated time periods. Whole cell lysates were then probed for p-ERK. (b) The bands were quantified, and the relative density of the Ctrl group was set to $1(\mathrm{n}=5$, ${ }^{*} \mathrm{P}<0.05$ vs. Ctrl; ${ }^{* *} \mathrm{P}<0.01$ vs. Ctrl). (c) Human podocytes were treated with or without HG for $24 \mathrm{~h}$ alone (Veh) or together with a $10 \mu \mathrm{M}$ PYR41 pretreatment for $30 \mathrm{~min}$. Whole cell lysates were then probed for p-ERK. (d) The bands were quantified, and the relative density of the Ctrl group was set to $1(\mathrm{n}=5, * \mathrm{P}$ $<0.05$ vs. Ctrl; " $\mathrm{P}<0.05$ vs. Veh).

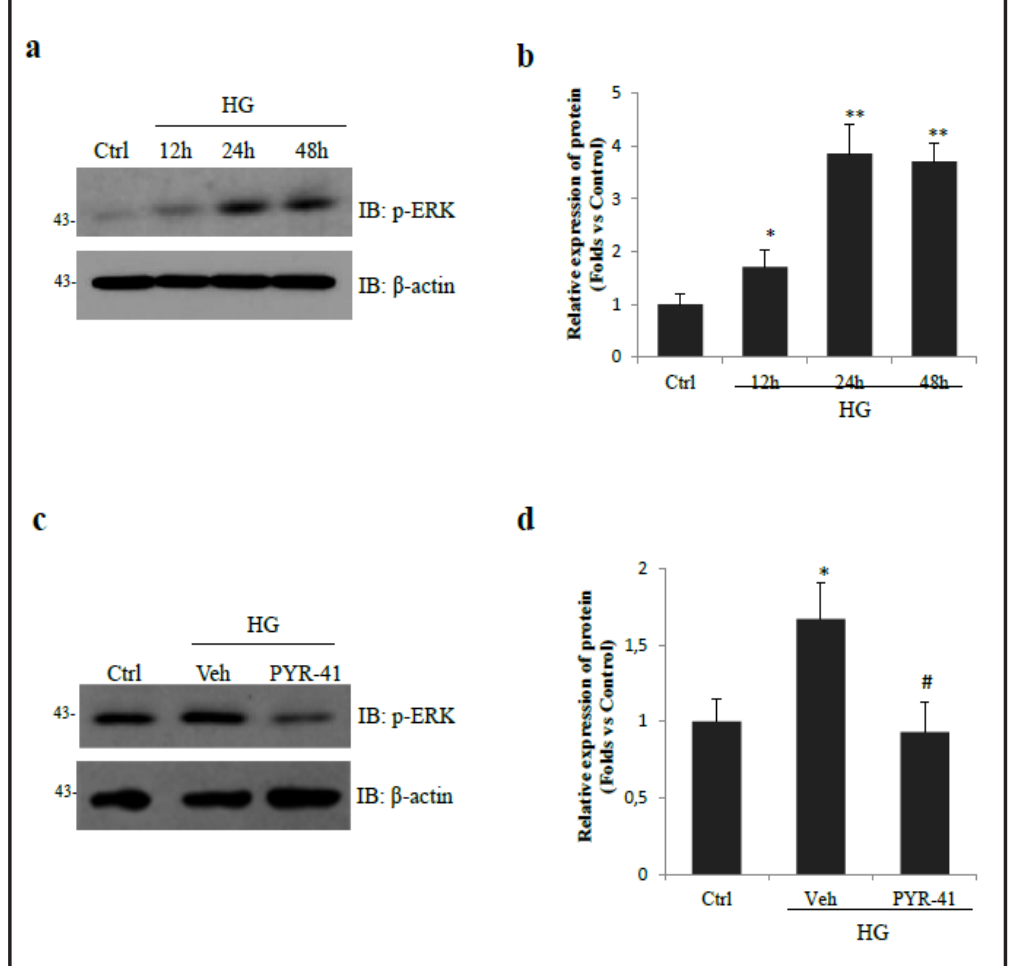




\section{Cellular Physiology \begin{tabular}{ll|l} 
DOI: 10.1159/000477329 & O 2017 The Author(s). Published by S. Karger AG, Basel \\
www.karger.com/cpb
\end{tabular} \\ Lei et al.: PKC- $\alpha$-EGFR-ERK Axis Involves in High Glucose Induced Podocyte Injury}

Fig. 6. Inhibition of the ubiquitin E1 enzyme minimizes HG-induced podocyte injury. (a) Human podocytes were stimulated with or without HG for the indicated time periods; then, the whole cell lysates were probed for desmin. (b) The bar graph shows the quantification of the desmin signals. The relative density of the Ctrl group was set to $1\left(\mathrm{n}=4,{ }^{*} \mathrm{P}\right.$ $<0.05$ vs. Ctrl). (c) Human podocytes were incubated with or without HG for $24 \mathrm{~h}$ alone (Veh) or together with a $10 \mu \mathrm{M}$ PYR-41 pretreatment for $30 \mathrm{~min}$. Whole cell lysates were then probed for desmin. (d) Densitometry analysis of desmin. ( $\mathrm{n}=5$, *P $<0.05$ vs. Ctrl; ${ }^{\#} \mathrm{P}<0.05$ vs. Veh). (e) Human podocytes were treated with or without HG for $24 \mathrm{~h}$ alone (Veh) or together with a $10 \mu \mathrm{M}$ PYR41 pretreatment for $30 \mathrm{~min}$. Cells were then stained with rhodamine-phalloidin to visualize F-actin. (f) The percentages of cells with distinct, longitudinal F-actin fibers are summarized. Scoring was determined based on 80 podocytes from each slide $(\mathrm{n}=3, * \mathrm{P}<0.05$ vs. Ctrl; " $\mathrm{P}<0.05$ vs. Veh). a

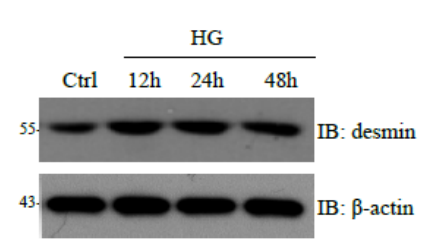

b

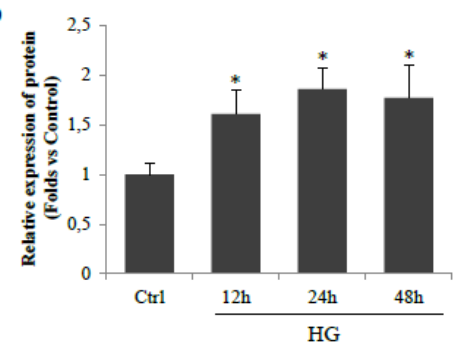

c

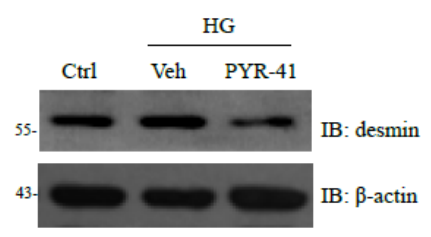

d
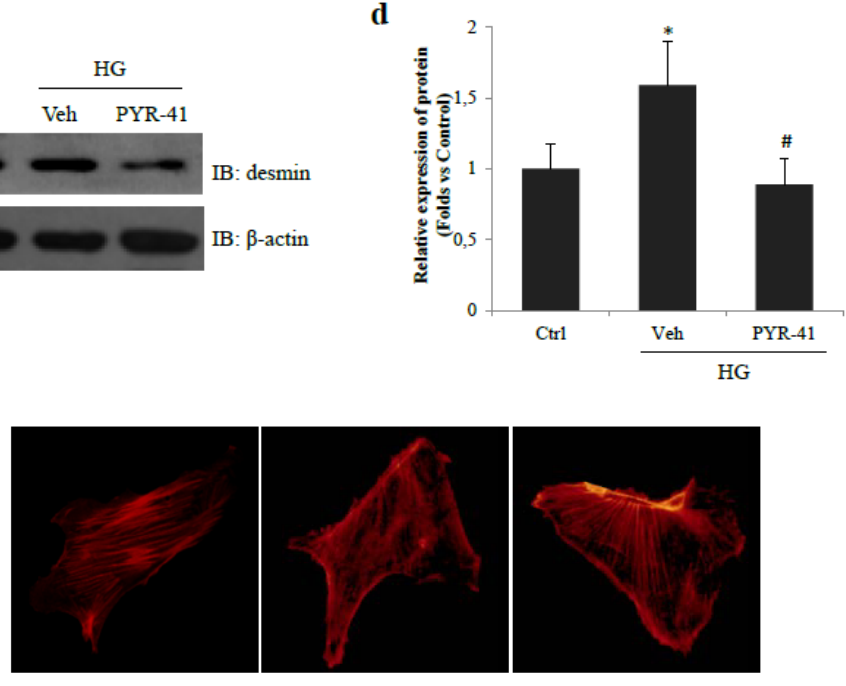

Ctrl

Veh PYR-41

HG

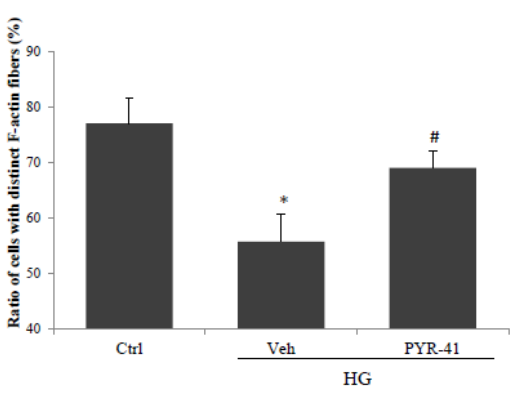

leakage of proteins into Bowman's capsules. It is well established that structural alterations and/or a depletion of podocytes are the core events and predictors of the progression of DKD [25-27]. Therefore, in this report, we addressed the potential pathogenesis of podocyte impairment during hyperglycemia.

During hyperglycemia, diacylglycerol is chronically upregulated, leading to sustained PKC activation, which contributes to the progression of DKD in multiple ways [26]. These effects include increased levels of prostaglandin E2 and nitric oxide, resulting in glomerular hyperfiltration [28, 29]; activated VEGF signaling, accelerating the development of microalbuminuria [5, 30]; and upregulated CTGF and TGF- $\beta$ levels, leading to the subsequent overproduction of fibronectin and type IV collagen, which contributes to glomerular basement membrane (GBM) thickening and ECM deposition [30].

The PKC protein family is divided into at least 12 subtypes, and PKC- $\alpha$ is believed to be the most critical in relation to DKD. However, evidence for the PKC- $\alpha$-related impairment of 


\section{Cellular Physiology Cell Physiol Biochem 2017;42:281-294 \\ \begin{tabular}{l|l} 
DOI: 10.1159/000477329 & Ond Biochemistry 2017 The Author(s). Published by S. Karger AG, Basel \\
www.karger.com/cpb
\end{tabular} \\ Lei et al.: PKC- $\alpha$-EGFR-ERK Axis Involves in High Glucose Induced Podocyte Injury}

podocytes, the key cell type involved in DKD, is rather limited. Only a few studies, most from the same group, have shown that podocytic PKC- $\alpha$ mediates nephrin and TGF- $\beta$ receptor I endocytosis, ultimately leading to podocyte injury [31-33]. Further exploration of the mechanisms of PKC- $\alpha$-related podocyte loss during hyperglycemia is important for the development of novel and potent therapeutic targets.

It is well recognized that PKC- $\alpha$ regulates EGFR signaling during malignant cell proliferation, and EGFR is ubiquitously expressed in various cells of the body. In the kidney, it is present in both tubule and glomerular cells, including podocytes [34-36]. Furthermore, EGFR has been verified to participate in crescentic nephritis, obstructive nephropathy, acute kidney injury, and renal fibrosis, as well as DKD [10, 37-42]. Following its activation, EGFR recruits adaptor proteins and triggers signaling cascades, which are implicated in diverse pathophysiological events [43]. Activated EGFR undergoes rapid internalization from the cell surface, and the activity of EGFR kinase is sustained following endocytosis. Indeed, internalization is required for the full activation of some membranous signaling molecules, such as G protein-coupled receptors, nerve growth factor receptors, and Notch [43]. Of note, the ubiquitin conjugation of channels and membranous receptors, including EGFR, has emerged as a key regulatory mechanism of endocytosis, post-endocytotic sorting, and the turnover of membrane proteins [44-48]. Thus, the ubiquitination and subcellular trafficking of EGFR are tightly linked to its downstream signaling activity and to the events this signaling controls.

Based on the involvement of both PKC- $\alpha$ and EGFR during DKD and the well-established connection of PKC- $\alpha$ with EGFR in malignancy, here we evaluated whether PKC- $\alpha$ regulates the endocytosis of EGFR and the subsequent signal transduction in an ubiquitination-dependent manner during podocyte injury in diabetes. Using immunoprecipitation, biotinylation and MesNa treatment, we found that under HG conditions, PKC- $\alpha$ accumulates on the cell surface, which accounts for the subsequent ubiquitination and internalization of EGFR.

Accumulating evidence indicates that PKC can mediate membranous protein ubiquitination and affect its trafficking [49-52]; however, this matter remains disputed, potentially due to the different disease models, pathogenic factors and other elements that have been tested [53-55]. EGFR internalization has been shown to be controlled by both ubiquitin-dependent and ubiquitin-independent mechanisms [56]. Furthermore, the ubiquitination of EGFR plays different roles in its internalization and post-endocytic trafficking at least in a cell type- and pathogenic stimuli-dependent manner [57]. Here, by pretreatment with the PKC- $\alpha$ antagonist Gö6976, we revealed that in HG-stimulated podocytes, PKC- $\alpha$ triggers EGFR ubiquitination and its ensuing internalization from the cell surface.

Moreover, our data elucidated that the blocking of ubiquitin ligation by PYR- 41 inhibits EGFR internalization and ERK phosphorylation and eventually prevents podocyte injury caused by hyperglycemia.

It is well known that ubiquitin-triggered EGFR internalization affects downstream signaling dynamics and consequently regulates cellular events. There is a well-described association between EGFR endocytosis and ERK phosphorylation in some cell lines. For example, Lazzara MJ and Vieira AV reported that the expression of a dominant negative form of dynamin (K44A) reduces EGFR internalization and markedly suppresses EGFRmediated ERK activation in lung cell lines and the immortal HeLa cervical cancer cell line $[58,59]$. Consistent with these findings, our results illustrate that in podocyte cell lines, EGFR endocytosis is associated with the subsequent cascade of ERK activation in HG-treated podocyte.

The expression of the EGFR ligand and its receptor are increased in DKD [41, 42]. Chen $\mathrm{J}$ et al [60] recently revealed that in STZ-induced type 1 diabetes, podocyte-specific EGFR-knockout mice exhibit significantly reduced albuminuria and less podocyte depletion than their wild-type littermates. All of these observations suggest that the EGFR pathway is actively involved in the pathogenesis of DKD. Accordingly, our findings indicate that endocytosis of EGFR and activation of its associated downstream pathway contributes to 


\section{Cellular Physiology Cell Physiol Biochem 2017;42:281-294 \begin{tabular}{l|l} 
DOI: 10.1159/000477329 & Ond 2017 The Author(s). Published by S. Karger AG, Basel \\
www.karger.com/cpb
\end{tabular} \\ Lei et al.: PKC- $\alpha$-EGFR-ERK Axis Involves in High Glucose Induced Podocyte Injury}

podocyte damage during DKD, which is regulated by the PKC- $\alpha$-mediated ubiquitination and trafficking of EGFR. Thus, blocking PKC- $\alpha$-associated EGFR signal transduction is a promising intervention for the prevention of podocyte damage during DKD.

Our study has several limitations. First, our findings suggest PKC- $\alpha$ is involved in EGFR ubiquitination and internalization from the cell surface; however, we solely use the ubiquitinactivating enzyme E1 inhibitor to test the connection between ubiquitination of EGFR and impairment of podocytes. Mutation of ubiquitination sites of EGFR will be adopted in our future studies to illustrate a more accurate link among ubiquitination, endocytosis and podocyte injury. Second, the protein trafficking data were primarily obtained from cultured podocytes, and further in vivo evidence is required to verify these results. Third, the postendocytotic sorting of membranous proteins is crucial for downstream signaling activity, and further investigation into the intracellular trafficking of EGFR is required. Fourth, cytokines, kinases and growth factors comprise a sophisticated network, and PKC- $\alpha$ and EGFR are only two of the major components of this network of diverse mediators involved in DKD. Thus, we should interpret our findings cautiously and with a broad perspective. Lastly, the phenotype of damaged podocytes varies at different stages of DKD; thus, future studies need to analyze these processes at different time points to assess the role of PKC- $\alpha$ and EGFR during the entire course of DKD.

Taken together, our new findings suggest that enhanced membranous PKC- $\alpha$ contributes to EGFR ubiquitination, endocytosis, and ERK activation, which eventually lead to podocyte damage under hyperglycemic conditions. Despite its limitations, the current study advances our understanding of the pathogenesis of podocyte injury in diabetes and provides clues for exploring new strategies for the treatment of DKD.

\section{Acknowledgements}

This work was supported by grants from the National Natural Science Foundation of China (No.31200872, No. 81570671, No. 81470964, and No. 81522010), a grant from Wuhan Science and Technology Bureau (No. 2015060101010039), and a Doctoral Fund from the Ministry of Education of China (No. 20130142110064).

\section{Disclosure Statement}

The authors declare that there are no conflicts of interest regarding the publication of this paper.

\section{References}

1 Craven PA, Derubertis FR: Protein kinase C is activated in glomeruli from streptozotocin diabetic rats. Possible mediation by glucose. J Clin Invest 1989;83:1667-1675.

2 Lee TS, Saltsman KA, Ohashi H, King GL: Activation of protein kinase C by elevation of glucose concentration: proposal for a mechanism in the development of diabetic vascular complications. Proc Natl Acad Sci U S A 1989;47:e11-16.

3 Matthias M, Jan M, Joon-Keun P, Hermann H: Nailing down PKC isoform specificity in diabetic nephropathy two's company, three's a crowd. Nephrol Dial Transplant 2007;22:2421-2425.

-4 Ibrahim S, Rashed L, Fadda S: Evaluation of renal gene expression of protein kinase C (PKC) isoforms in diabetic and nondiabetic proliferative glomerular diseases. Scientific World Journal 2008;8:835-844.

-5 Jan M, Joon-Keun P, Martin B, Marlies E, Carsten L, Torsten K, Matthias M, Faikah G, Annette F, Bahlmann FH: Diminished loss of proteoglycans and lack of albuminuria in protein kinase C-alpha-deficient diabetic mice. Diabetes 2004;53:2101-2109. 


\section{Cellular Physiology Cell Physiol Biochem 2017;42:281-294 \begin{tabular}{l|l} 
DOI: 10.1159/000477329 & O 2017 The Author(s). Published by S. Karger AG, Basel \\
www.karger.com/cpb
\end{tabular} \\ Lei et al.: PKC- $\alpha$-EGFR-ERK Axis Involves in High Glucose Induced Podocyte Injury}

6 Menne J, Meier M, J-K P, Boehne M, Kirsch T, Lindschau C, Ociepka R, Leitges M, Rinta-Valkama J, Holthofer $\mathrm{H}$ : Nephrin loss in experimental diabetic nephropathy is prevented by deletion of protein kinase $\mathrm{C}$ alpha signaling in-vivo. Kidney Int 2006;70:1456-1462.

7 Matthias M, Joon-Keun P, Daniel O, Torsten K, Carsten L, Faikah G, Michael L, Jan M, Hermann H: Deletion of protein kinase C-beta isoform in vivo reduces renal hypertrophy but not albuminuria in the streptozotocininduced diabetic mouse model. Diabetes 2007;56:346-354.

8 Citri A, Yarden Y: EGF-ERBB signalling: towards the systems level. Nat Rev Mol Cell Biol 2006;7:505-516.

-9 Thorner PS, Michael H, Vera E, Yoshikazu S, Susan Q: Podocytes contribute to the formation of glomerular crescents. J Am Soc Nephrol 2008;19:495-502.

10 Guillaume B, Martin F, Sandra S, Cécile F, Elisabeth R, Marine M, Eric S, Nathalie S, Sophie V, Ariane G: Epidermal growth factor receptor promotes glomerular injury and renal failure in rapidly progressive crescentic glomerulonephritis. Nat Med 2011;17:1242-1250.

11 Eden ER, Huang F, Sorkin A, Futter CE: The role of EGF receptor ubiquitination in regulating its intracellular traffic. Traffic 2012;13:329-337.

12 Simona P, Pier Paolo DF, Sara S: Keeping EGFR signaling in check: ubiquitin is the guardian. Cell cycle 2014;13:681-682.

13 Henrik O, Michael L: Protein kinase C alpha but not PKCzeta suppresses intestinal tumor formation in ApcMin/+ mice. Cancer Res 2006;66:6955-6963.

14 Xiao-Qi W, Qiu Y, Ping S, Ji-Wei L, Linda G, Mcdaniel SM, Paller AS: Suppression of epidermal growth factor receptor signaling by protein kinase $\mathrm{C}$-alpha activation requires CD82, caveolin-1, and ganglioside. Cancer Res 2007;67:9986-9995.

15 Karmakar P, Chakrabarti MK: Thermostable direct hemolysin diminishes tyrosine phosphorylation of epidermal growth factor receptor through protein kinase C dependent mechanism. Biochim Biophys Acta 2012;1820:1073-1080.

-16 Stewart JR, O'Brian CA: Protein kinase c- $\alpha$ mediates epidermal growth factor receptor transactivation in human prostate cancer cells. Mol Cancer Ther 2005;4:726-732.

17 Su H, Liu B, Fröhlich O, Ma H, Sands JM, Chen G: Small GTPase Rab14 down-regulates UT-A1 urea transport activity through enhanced clathrin-dependent endocytosis. FASEB J 2013;27:4100-4107.

18 Hua S, Qiang W, Xiu-Juan T, Fang-Fang H, Pan G, Hui T, Chen Y, Di F, Shan C, Yu-Mei W: MAD2B contributes to podocyte injury of diabetic nephropathy via inducing cyclin B1 and Skp2 accumulation. Am J Physiol Renal Physiol 2015;308:728-736.

19 Chen RX, Liu F, Li Y, Liu GA: Neuromedin S increases L-type Ca(2+) channel currents through G(i)alphaprotein and phospholipase C-dependent novel protein kinase $\mathrm{C}$ delta pathway in adult rat ventricular myocytes. Cell Physiol Biochem 2012;30(3):618-30.

-20 Wang L, Balzer MS, Rong S, Menne J, von Vietinghoff S, Dong L, Gueler F, Jang MS, Xu G, Timrott K, Tkachuk S, Hiss M, Haller H, Shushakova N: Protein kinase c alpha inhibition prevents peritoneal damage in a mouse model of chronic peritoneal exposure to high-glucose dialysate. Kidney Int 2016;89:1253-1267.

21 Su H, Carter CB, Laur O, Sands JM, Chen G: Forskolin stimulation promotes urea transporter UT-A1 ubiquitination, endocytosis, and degradation in MDCK cells. Am J Physiol Renal Physiol 2012;303:28-34.

22 Yang L, Zheng S, Epstein PN: Metallothionein over-expression in podocytes reduces adriamycin nephrotoxicity. Free Radic Res 2009;43:174-182.

23 Gao P, Meng XF, Su H, He FF, Chen S, Tang H, Tian XJ, Fan D, Wang YM, Liu JS, Zhu ZH, Zhang C: Thioredoxininteracting protein mediates nalp3 inflammasome activation in podocytes during diabetic nephropathy. Biochim Biophys Acta 2014;1843:2448-2460.

24 Chen T, Zheng LY, Xiao W, Gui D, Wang X, Wang N: Emodin ameliorates high glucose induced-podocyte epithelial-mesenchymal transition in-vitro and in-vivo. Cell Physiol Biochem 2015;35:1425-436

25 Xia S, Liu Y, Li X, Thilo F, Tepel M: Insulin Increases Expression of TRPC6 Channels in Podocytes by a Calcineurin-Dependent Pathway. Cell Physiol Biochem 2016;38:659-669.

26 Toyoda M, Najafian B, Y, Caramori M, Mauer M: Podocyte detachment and reduced glomerular capillary endothelial fenestration in human type 1 diabetic nephropathy. Diabetes 2007;56:2155-2160.

-27 Meyer TW, Bennett PH, Nelson RG: Podocyte number predicts long-term urinary albumin excretion in Pima Indians with Type II diabetes and microalbuminuria. Diabetologia 1999;42:1341-1344.

28 Bank N, Aynedjian HS: Role of EDRF (nitric oxide) in diabetic renal hyperfiltration. Kidney Int 1993;43:1306-1312. 


\section{Cellular Physiology Cell Physiol Biochem 2017;42:281-294 \begin{tabular}{l|l} 
DOI: 10.1159/000477329 & O 2017 The Author(s). Published by S. Karger AG, Basel \\
www.karger.com/cpb
\end{tabular} \\ Lei et al.: PKC- $\alpha$-EGFR-ERK Axis Involves in High Glucose Induced Podocyte Injury}

29 Williams B, Schrier RW: Glucose-induced protein kinase C activity regulates arachidonic acid release and eicosanoid production by cultured glomerular mesangial cells. J Clin Invest 1994;92:2889-2896.

30 Noh H, King GL: The role of protein kinase C activation in diabetic nephropathy. Kidney Int Suppl 2007;72:S49-53.

-31 Ivo Q, Magdalena W, Potthoff SA, Romy P, Eva KN, Sema S, Mario S, Johannes S, Oliver V, Lars Christian R: PKC alpha mediates beta-arrestin2-dependent nephrin endocytosis in hyperglycemia. J Biol Chem 2011;286:12959-12970.

-32 Irini T, Beina T, Jan M, Nelli S, Joon-Keun P, Becker JU, Friedrich M, Michael L, Hermann H, Mario S: Podocytic PKC-alpha is regulated in murine and human diabetes and mediates nephrin endocytosis. PloS one 2012;5:e10185.

-33 Kruger J, Meier M, Leitges M, Schiffer M, Tossidou I, Starker G, Haller H: PKC-alpha modulates TGF-beta signaling and impairs podocyte survival. Cell Physiol Biochem 2009;24:627-634.

-34 Harris DC, Chan L, Schrier RW: Remnant kidney hypermetabolism and progression of chronic renal failure. Am J Physiol 1988;254:F267-F276.

-35 Harris RC: Response of rat inner medullary collecting duct to epidermal growth factor. Am J Physiol 1989;256:F1117-F1124.

-36 Breyer MD, Redha R, Breyer JA: Segmental distribution of epidermal growth factor binding sites in rabbit nephron. Am J Physiol 1990;259:F553-F558.

-37 Jianchun C, Jian-Kang C, Kojiro N, David P, Mingqi T, Tang-Cheng L, Threadgill DW, Neilson EG, Harris RC: EGFR signaling promotes TGF $\beta$-dependent renal fibrosis. J Am Soc Nephrol 2012;23:215-224.

-38 Tang J, Liu N, Tolbert E, Ponnusamy M, Ma L, Gong R, Bayliss G, Yan H, Zhuang S: Sustained activation of EGFR triggers renal fibrogenesis after acute kidney injury. Am J Pathol 2013;17:160-172.

-39 Paizis K, Kirkland G, Polihronis M, Katerelos M, Kanellis J, Power DA: Heparin-binding epidermal growth factor-like growth factor in experimental models of membranous and minimal change nephropathy. Kidney Int 1998;53:1162-1171.

40 Tomoki M, Fenghua Z, Suwan W, Xiaofeng F, Huifang C, Haichun Y, Aihua B, Fogo AB, Harris RC: Low nitric oxide bioavailability upregulates renal heparin binding EGF-like growth factor expression. Kidney Int 2013;84:1176-1188.

41 Lee YJ, Shin SJ, Lin SR, Tan MS, Tsai JH: Increased expression of heparin binding epidermal growth-factorlike growth factor mRNA in the kidney of streptozotocin-induced diabetic rats. Biochem Biophys Res Commun 1995;207:216-222.

42 Sayedahmed N, Besbas N, Mundy J, Muchanetakubara E, Cope G, Pearson C, El NM: Upregulation of epidermal growth factor and its receptor in the kidneys of rats with streptozotocin-induced diabetes. Exp Nephrol 1996;4:330-339.

43 Alexander S, Mark VZ: Endocytosis and signalling: intertwining molecular networks. Nat Rev Mol Cell Biol 2009;10:609-622.

44 Manuel M, Wu CC, Tatiana S, Korstjens DR, Alexander S: Enhanced ubiquitylation and accelerated degradation of the dopamine transporter mediated by protein kinase C. J Biol Chem 2005;280:3561735624.

-45 Kumar KGS, Hervé B, Carbone CJ, Jianghuai L, Gayathri S, Ping X, Ying L, Baker DP, Junmin P, Lukacs GL: Sitespecific ubiquitination exposes a linear motif to promote interferon-alpha receptor endocytosis. J Cell Biol 2007;179:935-950.

46 Ruifeng Z, Patel SV, Snyder PM: Nedd4-2 catalyzes ubiquitination and degradation of cell surface ENaC. J Biol Chem 2007;282:20207-20212.

47 Varghese B, Barriere HCarbone CJ, Banerjee A, Swaminathan G, Plotnikov A, Xu P, Peng J, Goffin V, Lukacs GL, Fuchs SY: Polyubiquitination of prolactin receptor stimulates its internalization, postinternalization sorting, and degradation via the lysosomal pathway. Mol Cell Biol 2008;75:855-866.

48 Bomberger JM, Barnaby RL, Stanton BA: The deubiquitinating enzyme USP10 regulates the post-endocytic sorting of cystic fibrosis transmembrane conductance regulator in airway epithelial cells. J Biol Chem 2009;284:18778-18789.

49 Juan-Sanz JD, Zafra F, López-Corcuera B, Aragón C: Endocytosis of the neuronal glycine transporter GLYT2: role of membrane rafts and protein kinase C-dependent ubiquitination. Traffic 2011;12:1850-1867. 


\section{Cellular Physiology Cell Physiol Biochem 2017;42:281-294 \begin{tabular}{l|l} 
DOI: 10.1159/000477329 & Ond 2017 The Author(s). Published by S. Karger AG, Basel \\
www.karger.com/cpb
\end{tabular}}

Lei et al.: PKC- $\alpha$-EGFR-ERK Axis Involves in High Glucose Induced Podocyte Injury

50 Xu D, Wang H, Zhang Q, You G: Nedd4-2 but not Nedd4-1 is critical for protein kinase C-regulated ubiquitination, expression, and transport activity of human organic anion transporter 1. Am J Physiol Renal Physiol 2016;310:F821-F831.

51 Noemí GT, González-González IM, Jaime MV, Enrique FS, Cecilio G, Francisco Z: Protein kinase C (PKC)promoted endocytosis of glutamate transporter GLT-1 requires ubiquitin ligase Nedd4-2-dependent ubiquitination but not phosphorylation. J Biol Chem 2012;287:19177-19187.

52 Arnau VV, Julia BS, Tatiana S, Ellen Ildicho C, Alexander S: Protein kinase C-dependent ubiquitination and clathrin-mediated endocytosis of the cationic amino acid transporter CAT-1. J Biol Chem 2011;286:86978706.

53 Chandrasekaran P, Moore V, Buckley M, Spurrier J, Kehrl JH, Venkatesan S: HIV-1 Nef down-modulates $\mathrm{C}-\mathrm{C}$ and $\mathrm{C}-\mathrm{X}-\mathrm{C}$ chemokine receptors via ubiquitin and ubiquitin-independent mechanism. PloS one 2014;9:e86998.

54 Bolin LL, Hanson LK, Slater JS, Kerry JA, Campbell AE: Murine cytomegalovirus US22 protein pM140 protects its binding partner, pM141, from proteasome-dependent but ubiquitin-independent degradation. J Virol 2010;84:2164-2168.

55 Kumar KGS, Bentley V, Anamika B, Baker DP, Constantinescu SN, Sandra P, Fuchs SY: Basal ubiquitinindependent internalization of interferon alpha receptor is prevented by Tyk2-mediated masking of a linear endocytic motif. J Biol Chem 2008;283:18566-18572.

56 Fangtian H, Kuan GL, Alexander S: EGF receptor ubiquitination is not necessary for its internalization. Proc Natl Acad Sci U S A 2007;104:16904-16909.

-57 Huang F, Kirkpatrick DX, Gygi S, Sorkin A: Differential regulation of EGF receptor internalization and degradation by multiubiquitination within the kinase domain. Mol Cell 2006;21:737-748.

-58 Lazzara MJ, Lane KR, Jasper PJ, Yaffe MB, Sorger PK, Jacks T, Neel BG, Lauffenburger DA: Impaired SHP2mediated extracellular signal-regulated kinase activation contributes to gefitinib sensitivity of lung cancer cells with epidermal growth factor receptor-activating mutations. Cancer Res 2010;70:3843-3850.

59 Vieira AV, Lamaze C, Schmid SL: Control of EGF receptor signaling by clathrin-mediated endocytosis. Science 1996;274:2086-2089.

60 Chen J, Chen JK, Harris RC: EGF receptor deletion in podocytes attenuates diabetic nephropathy. J Am Soc Nephrol 2015;26:1115-1125. 